Research Article

\title{
Paraquat Exposure Induces Pulmonary Cell Mitophagy by Enhancing the PINK1/Parkin Signaling
}

\author{
Kaixiang Liu, ${ }^{1,2}$ Zhipeng Zhan, ${ }^{1}$ Wei Gao, ${ }^{1}$ Jie Feng, ${ }^{1}$ and Xisheng Xie ${ }^{1}{ }^{1}$ \\ ${ }^{1}$ Department of Nephrology, \\ The Second Clinical Medical Institution of North Sichuan Medical College (Nanchong Central Hospital), Nanchong 637000, \\ Sichuan Province, China \\ ${ }^{2}$ Department of Nephrology, The Chengdu Second Affiliated Hospital of Chongqing Medical University, \\ The Third People's Hospital of Chengdu, Chengdu 610031, China
}

Correspondence should be addressed to Xisheng Xie; xishengx@sina.com

Received 26 September 2019; Revised 3 December 2019; Accepted 11 December 2019; Published 22 January 2020

Academic Editor: Pankaj K. Bhavsar

Copyright (c) 2020 Kaixiang Liu et al. This is an open access article distributed under the Creative Commons Attribution License, which permits unrestricted use, distribution, and reproduction in any medium, provided the original work is properly cited.

\begin{abstract}
Paraquat (PQ) poisoning can cause oxidative stress, acute lung injury (ALI), and fibrosis. Excess oxidative stress can induce mitophagy. However, whether PQ exposure can induce mitophagy, contributing to the development of ALI and pulmonary fibrosis in vivo has not been clarified. Here, we show that PQ exposure induces ALI and fiber accumulation in the lung of rats in a time-dependent manner, accompanied by upregulating fibronectin (FN) and Collagen I (COL-I) expression. PQ exposure increased mitophagosome formation and PINK1 and Parkin expression in the lungs of rats. Similarly, PQ exposure reduced the viability and mitochondrial membrane potential, but enhanced FN, COL-I, PINK1, and Parkin expression in A549 cells. In contrast, PINK1 silencing significantly mitigated the PQ-upregulated Parkin, FN, and COL-I expression in A549 cells. Hence, PQ exposure induced ALI and fibrosis in rats by enhancing the PINK1/Parkin signaling and profibrotic factor expression in the lungs. Therefore, our findings suggest that the PINK1/Parkin signaling may be new therapeutic targets and may provide new insights in the pathogenesis of PQ-related ALI in rats.
\end{abstract}

\section{Introduction}

Paraquat (PQ) is a unique heterocyclic herbicide and widely used in many developing countries in the world. Chemically, PQ is an oxidant and its exposure may cause poisoning, such as acute respiratory distress syndrome (ARDS) and pulmonary fibrosis, by inducing systemic oxidative stress [1]. Unfortunately, patients with PQ poisoning have a high mortality because currently there is no specific antidote or effective therapy for PQ poisoning [2]. Pathophysiologically, PQ enters the body and can accumulate in pulmonary alveolar epithelial cells, which causes oxidative stress-related lung injury and progressive pulmonary fibrosis, leading to development of respiratory failure and multiple organ dysfunctional syndrome (MODS). Subacute PQ poisoning commonly causes progressive pulmonary fibrosis within days and weeks after exposure [3]. However, the pathogenesis of PQ-related progressive pulmonary fibrosis remains poorly understood.

Previous studies have shown that PQ produces numerous oxygen radicals through a series of linkage reactions when PQ enters the body, particularly in alveolar epithelial cells to form reactive oxygen species (ROS) [4]. The high levels of ROS produced by PQ can cause lipid peroxidation and alveolar epithelial cell apoptosis [5]. Furthermore, PQinduced pulmonary fibrosis can also be attributed to ROSinduced DNA damage, mitochondrial dysfunction, inflammation, and protease imbalance.

Mitochondrial autophagy (mitophagy) is defined as many factors (such as hypoxia, hunger, photosynthesis, oxidative stress, and others) stimulating the selective 

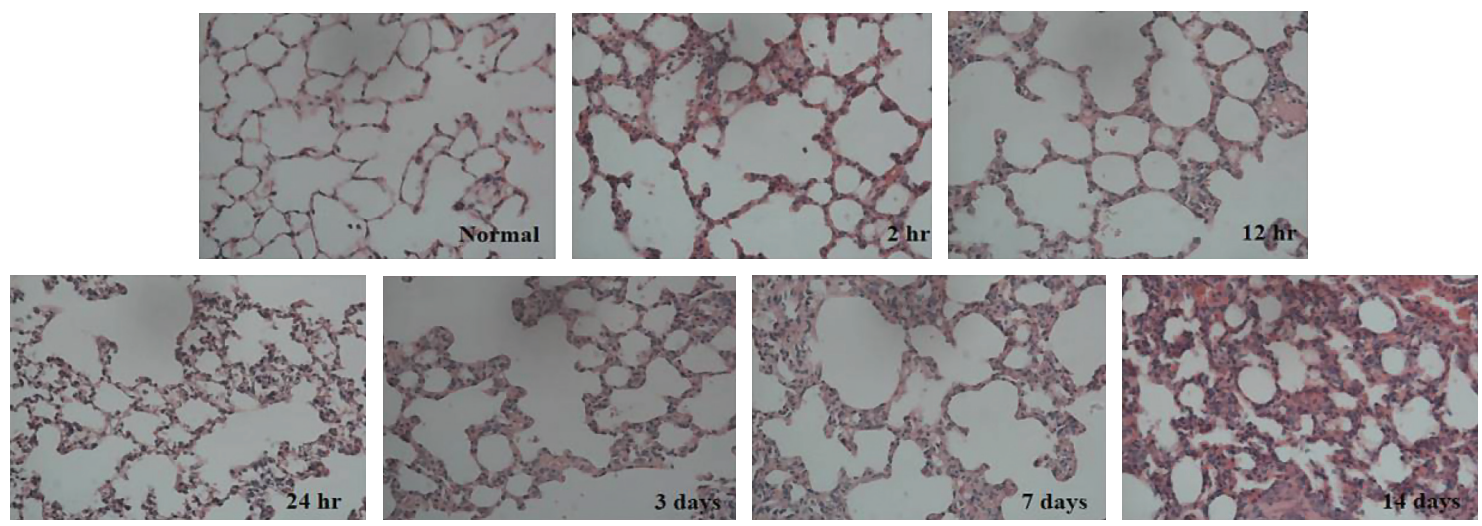

(a)
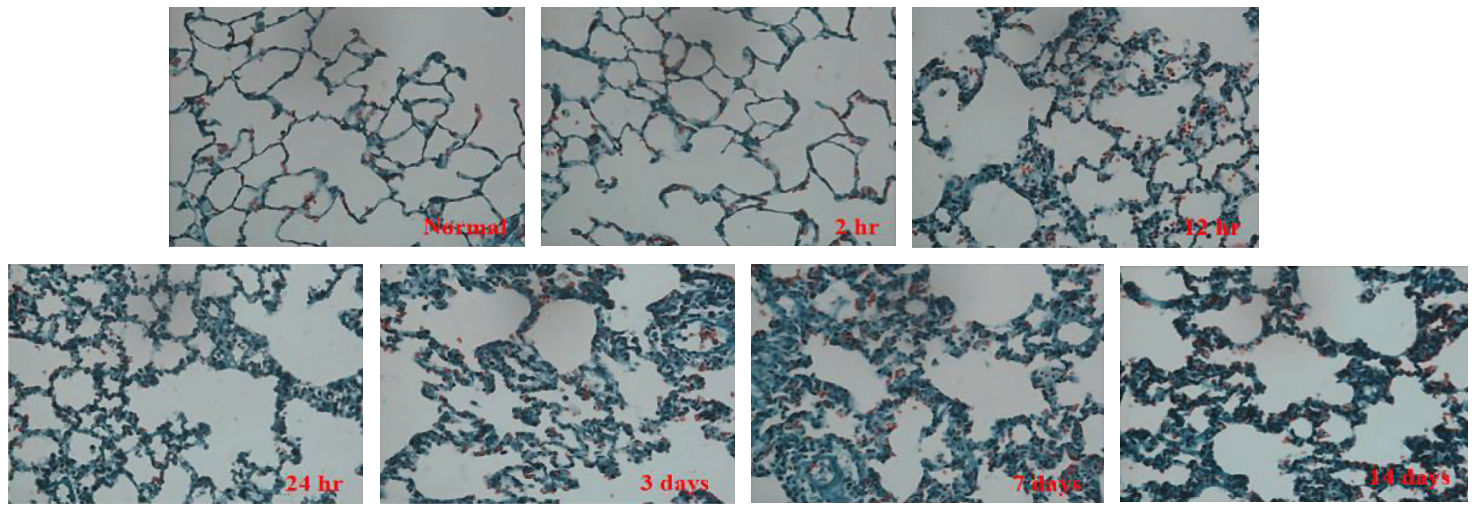

(b)

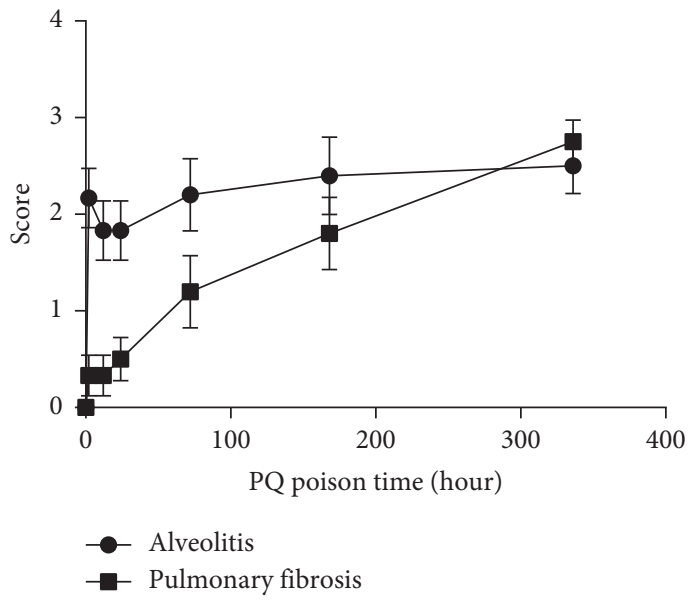

(c)

FIgURE 1: PQ exposure induces acute alveolitis and pulmonary fibrosis in rats. After PQ exposure, the rats were sacrificed at the indicated time points and their lung tissues were examined by H\&E and Masson's staining. The severities of alveolitis and pulmonary fibrosis in individual rats were scored. Data are representative images (magnification $\times 200$ ) or expressed as the mean \pm SD of each group $(n=5-6)$ from two separate experiments. (a) The H\&E staining. (b) Masson's trichrome staining. (c) The scores of alveolitis and pulmonary fibrosis in rats.

degradation of defect mitochondria [6]. During the process of mitophagy, the damaged, defective, and aged mitochondrial membrane is depolarized, which recruits the PTEN-induced kinase 1 (PINK1) and Parkin. Subsequently, PINK1 activates Parkin on the outer mitochondrial membrane and the defect mitochondria are wrapped into the phage specifically and degraded through the ubiquitination and lysosomal degradation processes.
PQ produces ROS, which damages the mitochondria, leading to altered mitochondrial membrane potential (MMP) and cell apoptosis. However, it is unknown whether PQ induces mitophagy and affects the PINK1/ Parkin signaling in alveolar epithelial cells and in the lung of rats.

In this study, we employed a rat model of PQ poisoning and A549 cells to investigate the effect of PQ exposure on the 


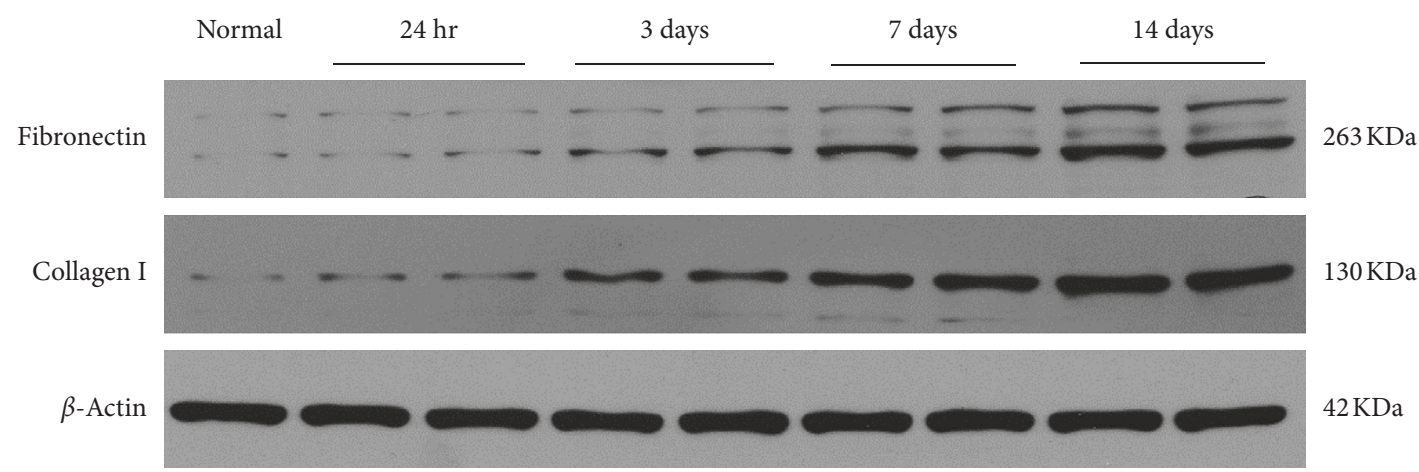

(a)

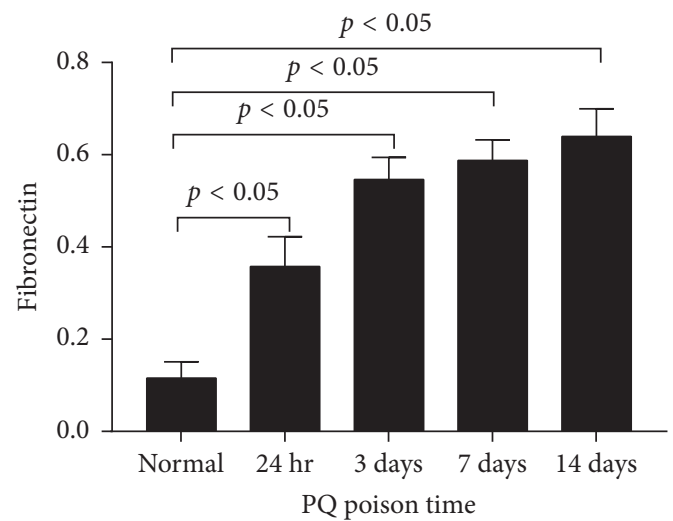

(b)

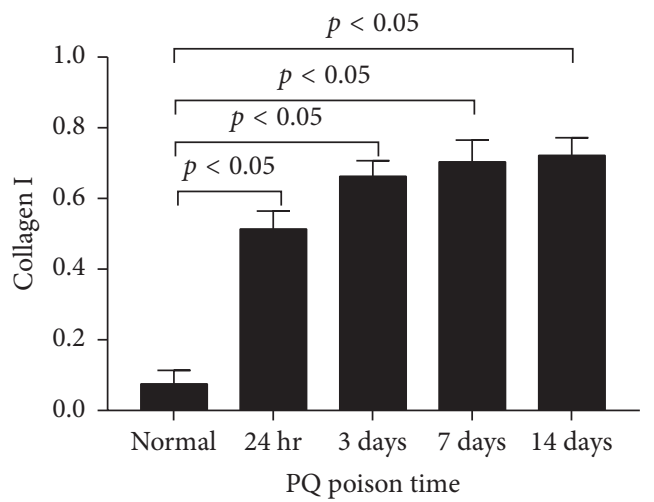

(c)

FIGURE 2: PQ poisoning enhances the FN and COL-I expression in the lungs of rats. The relative levels of FN and COL-I expression in the different groups of rats were determined by western blot. (a) Representative western blot images. (b, c) Quantitative analysis of FN and COLI expression. Data are representative images or expressed as the mean \pm SD of each group $(n=5-6)$ from three separate experiments.

dynamic changes in the lung injury and fibrosis as well as PINK1 and Parkin expression.

\section{Materials and Methods}

2.1. Materials and Instruments. The special reagents and equipment included a standard paraquat (Sigma-Aldrich, St. Louis, MO, USA); Cytoplasmic Protein Extraction Kit, Mitochondrial membrane potential assay kit with JC-1, and cell counting kit (C2006, Beyotime Biotechnology, Shanghai, China); antibodies anti-PINK1 (ab23707), antiParkin (ab77924), anti-Collagen I (ab34710), anti- $\beta$-actin (ab119716, abcam, Cambridge, UK), and anti-Fibronectin (66042-1-lg, Sanying, Wuhan, China); other analytical grade of chemical reagents (Aspen, Beijing, China); Flow cytometry (Beckman FC500, Indianapolis, Beckman); Upright Metallurgical Microscope (Nikon 80i, Tokyo, Japan); transmission electron microscope (Hitachi H-600IV, Tokyo, Japan).

2.2. Animals. Male Sprague Dawley (SD) rats at 6 weeks of age and $180-220 \mathrm{~g}$ body weight were obtained from the Experimental Animal Center of the North Sichuan Medical College. The rats were housed in a specific pathogen-free facility at consistent temperature of $20-25^{\circ} \mathrm{C}$ with a cycle of 12-h light/dark. Animals were allowed free-access to food and water ad libitum. The experimental protocol was approved by the Ethics Committee of the North Sichuan Medical College.

2.3. Animal Model and Grouping. The rats were randomized and provided with $5 \mathrm{ml}$ of saline (control, $n=6$ ) or $50 \mathrm{mg} / \mathrm{kg}$ PQ (a suitable dose, based on preliminary studies) in $5 \mathrm{ml}$ of saline by gavage (PQ group, $n=36$ ). At $2 \mathrm{~h}, 12 \mathrm{~h}, 1 \mathrm{~d}, 3 \mathrm{~d}, 7 \mathrm{~d}$, and $14 \mathrm{~d}$ after PQ administration, some rats were anesthetized ( $n=5-6$ per time point) and subjected to thoracotomy. The proximal end of the main bronchus and the distal end of the trachea were ligated. The left lung was washed three times with saline, and both lungs were collected. The left lung tissue (about $1 \mathrm{~mm}^{3}$ ) was biopsied from individual rats and fixed in $3 \%$ glutaraldehyde overnight. The remaining left lung of each rat was fixed in $4 \%$ paraformaldehyde at $4^{\circ} \mathrm{C}$ overnight and paraffin-embedded. The superior and meddle lubes of the right lungs in individual rats were immediately frozen in liquid nitrogen and stored at $-80^{\circ} \mathrm{C}$.

2.4. Histopathology. The left lung tissue sections $(3 \mu \mathrm{m})$ were deparaffined, rehydrated, and stained with haemotoxylin and eosin (H\&E) and Masson's trichrome. The sections were photoimaged under a light microscope, and the severities of 

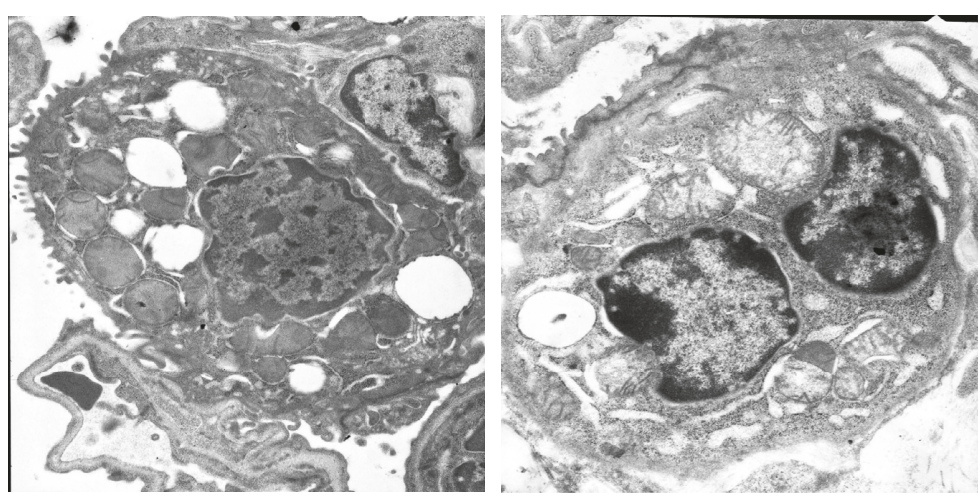

(a)

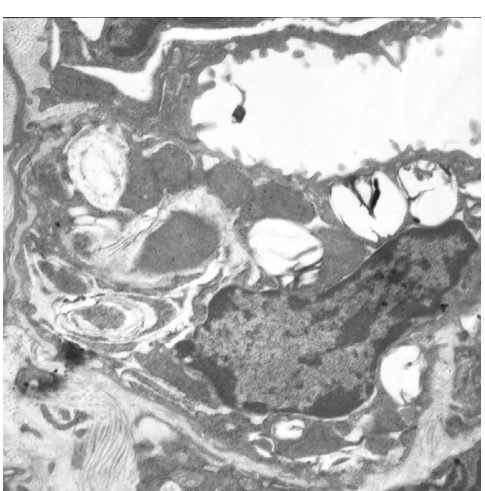

14 days

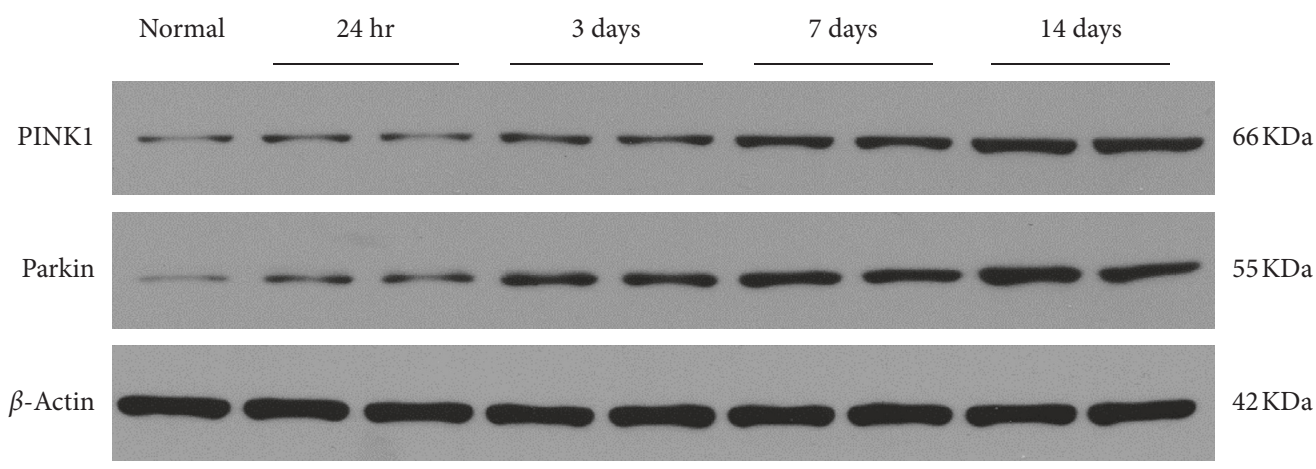

(b)
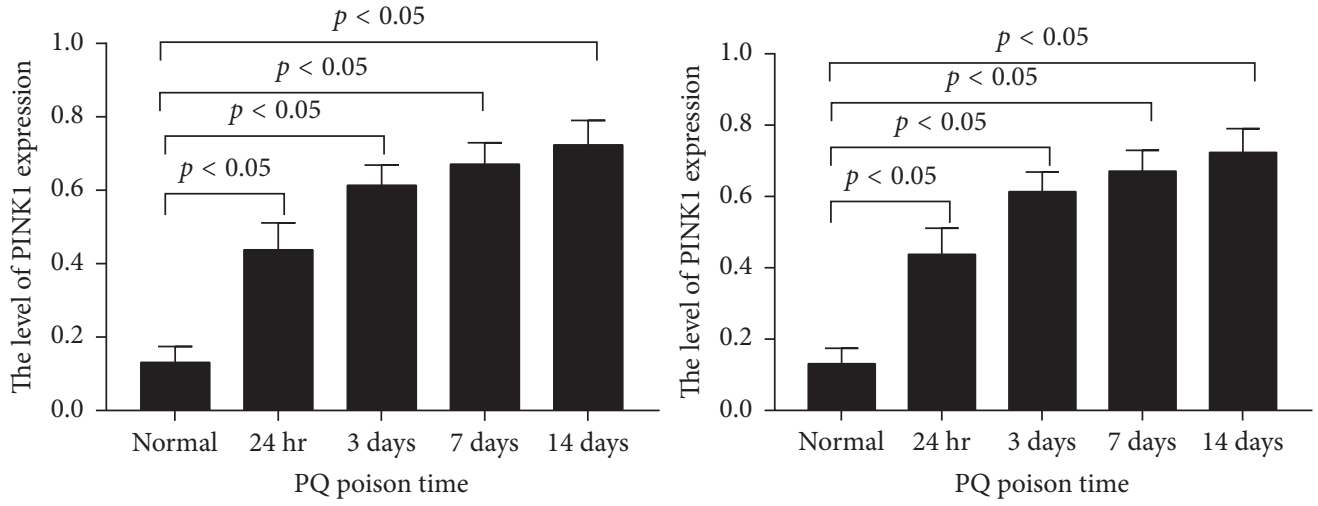

(c)

Figure 3: PQ exposure induces mitophagy in the lungs of rats. (a) TEM analysis indicates mitophagosome formation in the PQ-exposed lung epithelial cells. (b) Western blot analysis of PINK1 and Parkin expression in the lungs of rats. (c) Quantitative analysis of PINK1 and Parkin expression in the lungs of rats. Data are representative images or expressed as the mean \pm SD of each group $(n=5-6)$ from three separate experiments.

lung injury and fibrosis in ten visual fields of each section and five sections per animal were scored by pathologists in a blinded manner, as described previously [7].

\subsection{Detection of Mitochondrial Membrane Potential} (MMP) by Flow Cytometry. The different groups of cells were stained with JC-1 using the specific kit, according to the manufacturer's instruction, and the MMP in individual samples was analyzed by flow cytometry.

2.6. Western Blotting. The middle lobe of the right lung from individual rats was homogenized, and the mitochondrial proteins were extracted using a mitochondria protein extraction kit, according to the manufacturer's instructions. After determining the concentrations of total proteins by the BCA method, individual mitochondrial protein samples ( $15 \mu \mathrm{g} /$ lane) were separated by sodium dodecyl sulfate polyacrylamide gel electrophoresis on $10 \%$ gels and transferred onto polyvinylidene difluoride (PVDF) membranes. The membranes were blocked in 5\% nonfat dry milk in TBST and incubated with anti-PINK1, anti-Parkin, anti-FN, anti-COL-I, and anti- $\beta$-actin at $4^{\circ} \mathrm{C}$ overnight. After being washed, the bound antibodies were detected with horseradish peroxidase- (HRP-) conjugated secondary antibodies $(1: 500)$ at room temperature for $2 \mathrm{~h}$ and 


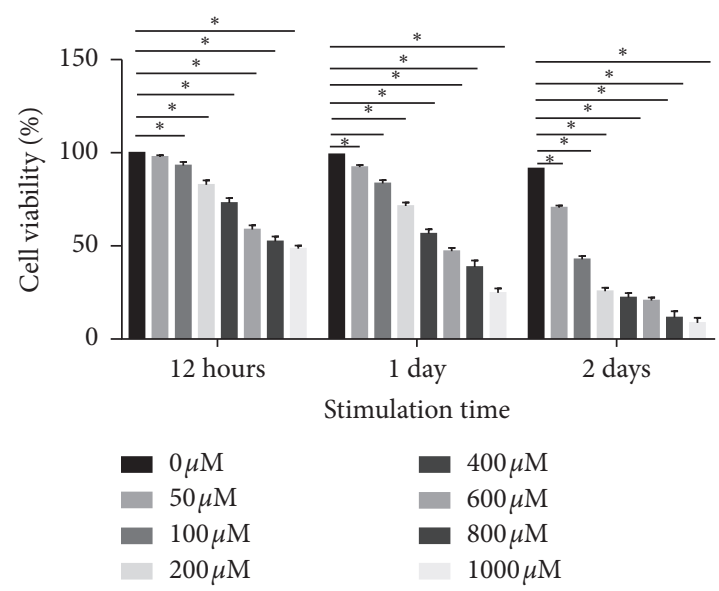

(a)
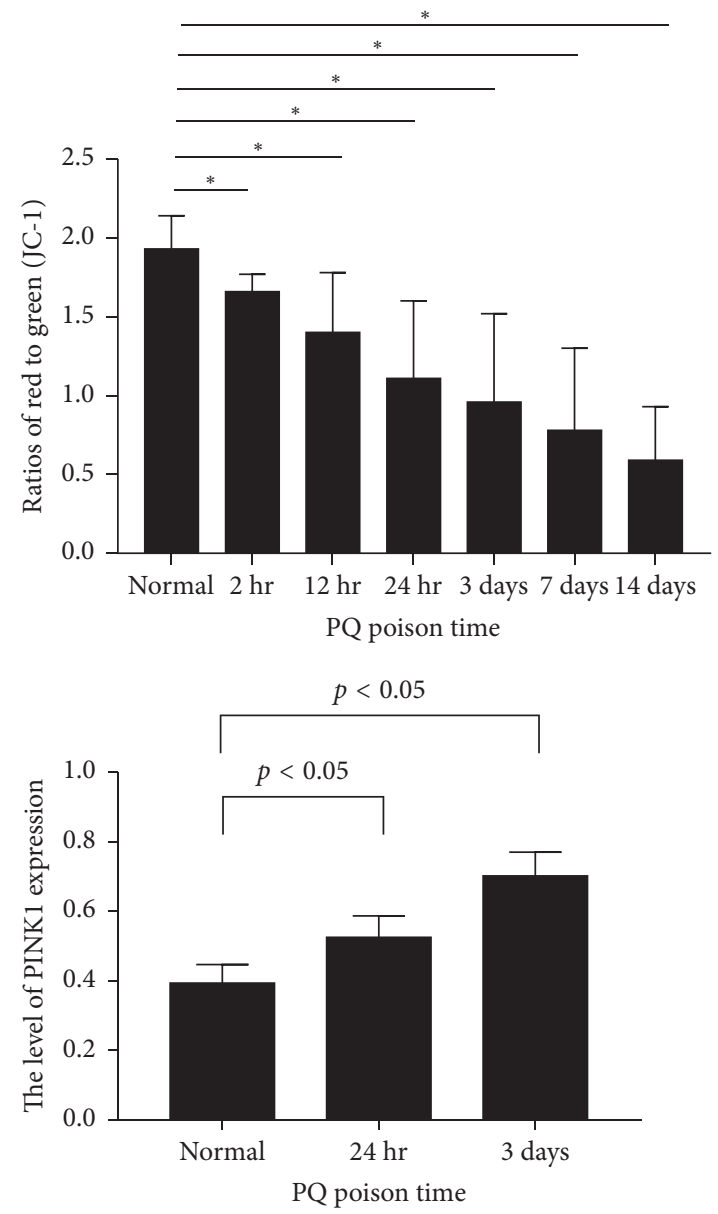

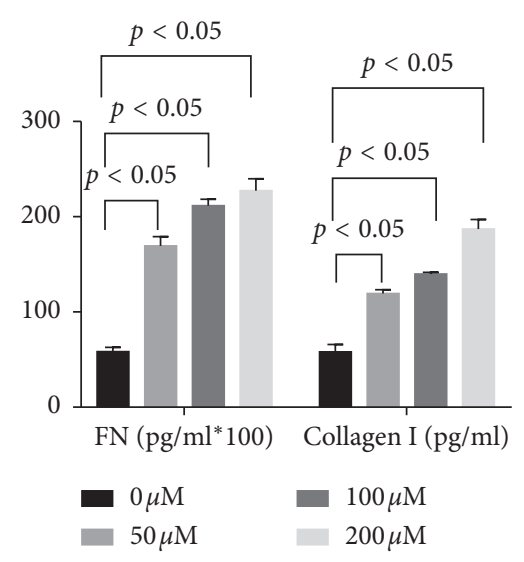

(b)
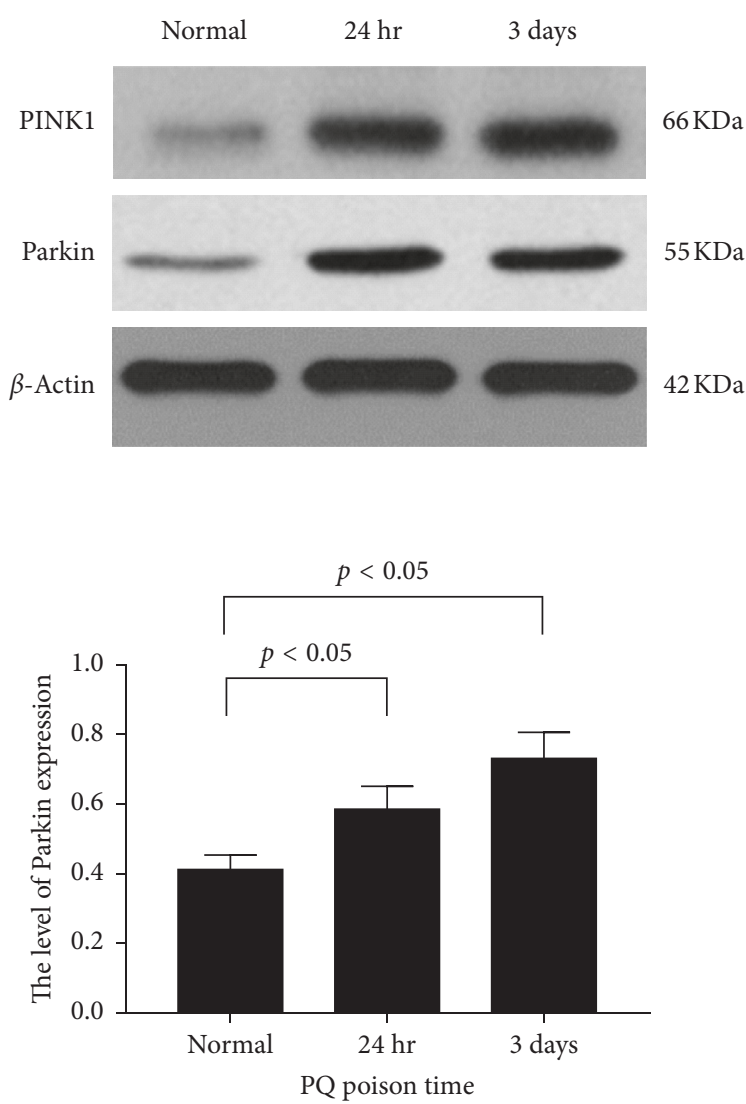

(c)

FIgURE 4: PQ exposure causes cytotoxicity against A549 cell by inducing mitophagy. (a) A549 cells were exposed in triplicate to PQ at the indicated doses for varying time periods and their viability was determined by CCK- 8 assays. (b) The relative levels of FN and COL-I in individual groups of cells were determined. (c) The MMP of each group of cells was examined by JC-1 staining and flow cytometry. (d) The relative levels of PINK1 and Parkin expression were examined by western blot. Data are representative images or expressed as the mean \pm SD of each group of cells from three separate experiments.

visualized using the enhanced chemiluminescent reagents. The relative levels of target proteins to the control $\beta$-actin were determined by densitometric analysis using the ImageJ software.
Similarly, the relative levels of PINK1, Parkin, FN, and COL-I to $\beta$-actin expression in the different groups of A549 cells were determined by western blot assay using specific antibodies. 


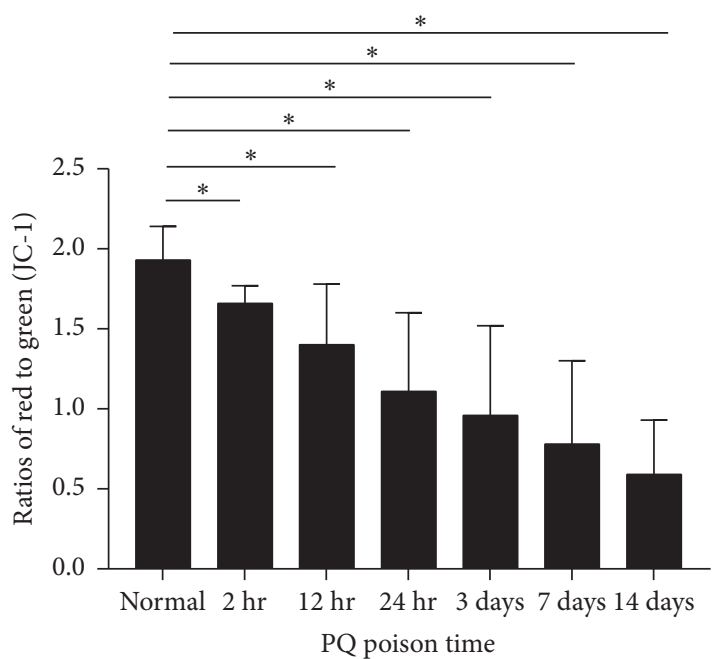

(a)

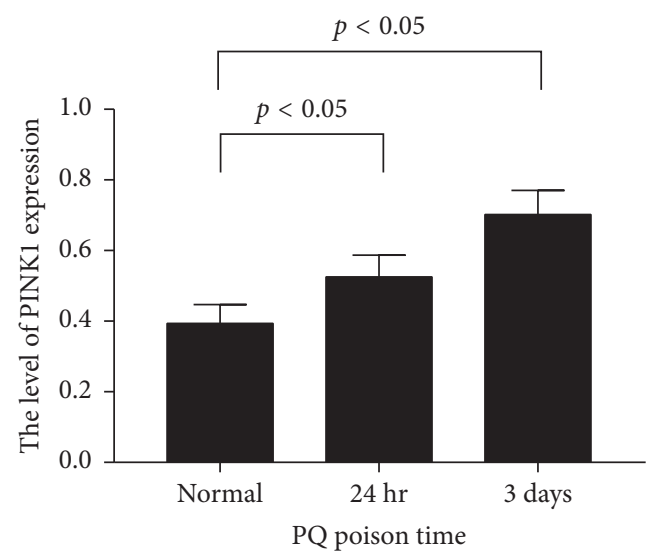

(c)

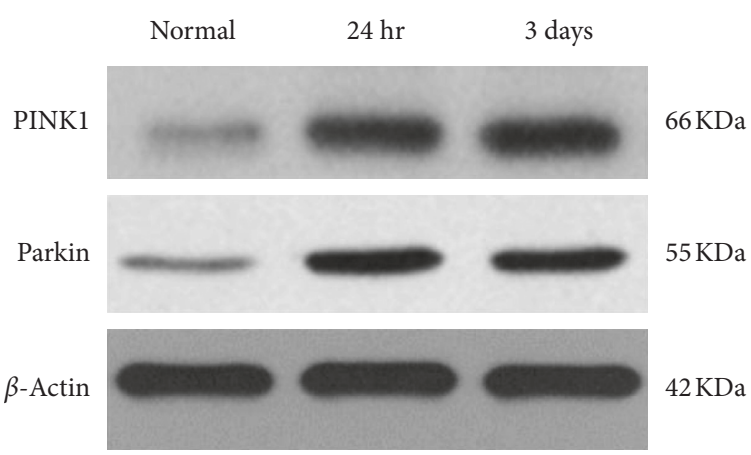

(b)

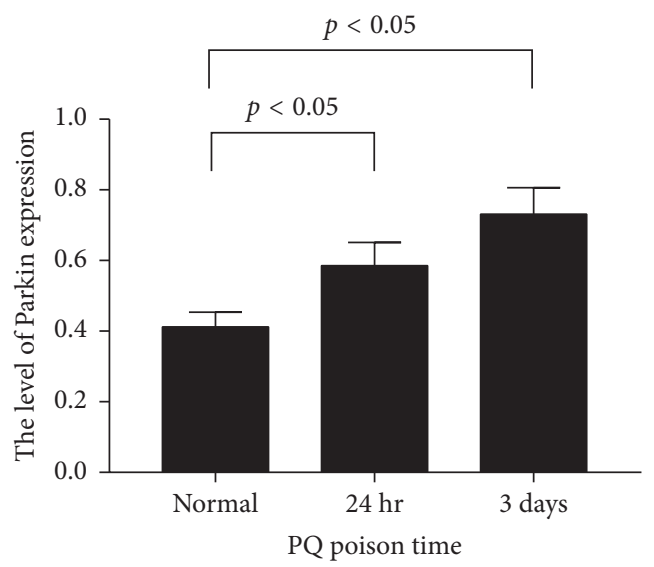

(d)

Figure 5: PINK1 silencing mitigates the PQ-upregulated FN, Col-I, and Parkin expression in A549 cells. A549 cells were transfected with control or PINK1-specific siRNA for $72 \mathrm{~h}$ and exposed to PQ for $24 \mathrm{~h}$. The relative levels of Parkin, FN, and COL-1 expression in individual groups of cells were quantified by western blot assays. Data are representative images or expressed as the mean \pm SD of each group of cells from three separate experiments.

2.7. Transmission Electron Microscopy. Individual lung tissue samples were prefixed with $3 \%$ glutaraldehyde, postfixed in $1 \%$ osmium tetroxide, dehydrated in acetone, infiltrated in Epox 812, and embedded. The lung tissue semithin sections $(50 \mathrm{~nm})$ were stained with uranyl acetate and lead citrate. The sections were examined using a transmission electron microscope.

2.8. Cell Culture. A549 cells were obtained from American Type Culture Collection (ATCC, Manassas, Virginia, USA) and cultured in DMEM medium supplemented with $10 \%$ fetal bovine serum (FBS) at $37^{\circ} \mathrm{C}$ in an incubator of $5 \% \mathrm{CO}_{2}$. When cells reached $60 \sim 70 \%$ of confluency in the logarithmic growth phase, the cells were used for subsequent experiments.

2.9. Gene, Plasmids, and Transfection. A549 cells were cultured in 24-well plates overnight and transfected with control siRNA (siN0000001-1-5) or PINK1-specific siRNA (stB0005003A-1-5, Ribo Biotechnologies, China) using the Lipofectamine 2000 (11668019, ThermoFisher Scientific) for three days and exposed to PQ. Twenty-four hours later, the cells were harvested, and the relative levels of Parkin, FN, and COL-I to $\beta$-actin expression in individual groups of cells were determined by Western blot.

2.10. Cell Viability. The cell viability was determined using the cell counting kit (CCK-8), according to the manufacturer's protocol. Briefly, A549 cells $\left(2 \times 10^{5} /\right.$ well $)$ were cultured in 96well plates overnight and treated in quintuplicate with vehicle saline or different concentrations $(50,100,200,400,600,800$, or $1000 \mu \mathrm{M})$ of PQ for varying periods (12-72 h). During the last 2 -h culture, individual wells were added with CCK- 8 solution $(10 \mu \mathrm{L})$ and the absorbance $(\mathrm{A})$ of individual wells was measured at $450 \mathrm{~nm}$. The wells without cells served as blank control. The cell viability was calculated using the equation: 


$$
\text { Cell viability }(\%)=\frac{\left[\left(A_{\text {paraquat }}-A_{\text {blank control }}\right)-\left(A_{\text {normal control }}-A_{\text {blank control }}\right)\right]}{\left(A_{\text {normal control }}-A_{\text {blank control }}\right)} \times 100 \% \text {. }
$$

2.11. Statistical Analysis. Data are expressed as mean$\mathrm{s} \pm$ standard deviation (SD). The difference among groups was analyzed by one-way analysis of variance (ANOVA), and the difference between groups was analyzed by Student's $t$ test using SPSS 17.0 (IBM, Armonk, NY, USA). A $p$ value $<0.05$ was considered statistically significant.

\section{Results}

3.1. PQ Causes Pulmonary Tissue Damage in Rats. To determine the effect of PQ on the lung injury and fibrosis, SD rats were randomized and provided with vehicle or PQ by gavage. The rats were sacrificed at different time points after PQ administration, and their lung tissues were examined by H\&E and Masson's trichrome staining. Following PQ exposure, four out of 36 rats died on day 3, 5, 9, and 12 after exposure. As shown in Figures 1(a) and 1(b), while there was normal alveolar structure in the control group of rats, there were moderate inflammation and collagen fibers surrounding the alveolar tissues. The alveolar inflammation and collagen fiber accumulation increased with time after PQ administration. On day 14 after PQ administration, there were many collagen fibers accumulated in the alveolar tissues, which disrupted the alveolar structure in rats. Semiquantitative analysis indicated that the severity of alveolitis and pulmonary fibrosis increased with time (Figure 1(c)). To look for additional evidence of fibrosis, the relative levels of FN and COL-I expression in the lungs of different groups of rats were determined by western blot. In comparison with those in the control rats, the levels of FN and COL-I expression in the lungs of rats following $\mathrm{PQ}$ exposure increased in a timedependent manner (Figure 2). Such data indicated that PQ exposure induced acute alveolitis and progressive pulmonary fibrosis in rats.

PQ exposure reduces the MMP and induces mitophagy by activating the PINK1/Parkin signaling in the lung of rats.

Oxidative stress can damage the mitochondria. To understand the consequence of $\mathrm{PQ}$-induced inflammation and fibrosis, we examined mitophagy in the lung cells from the indicated groups of rats by TEM (Figure 3(a)). In comparison with the control cells, there was obviously mitochondrial swelling in alveolar epithelial cells at $24 \mathrm{~h}$ after PQ exposure, and the degrees of mitochondrial swelling gradually increased with vacuoles in the mitochondria. Interestingly, there were denatured mitochondria surrounded by a vacuolar bilayer membrane-like structure, forming the typical double-layer wrapped mitophagy in alveolar epithelial cells from the PQexposed rats, but not in the control (Figure 3(a)). In addition, western blot analysis indicated that the relative levels of PINK1 and Parkin expression in the lungs from the PQexposed rats increased with time (Figures 3(b) and 3(c)). Collectively, such data indicated that PQ exposure induced mitophagy in alveolar epithelial cells by enhancing the PINK1/Parkin expression in the lung of rats.
3.2. PQ Reduces the Viability of A549 Cells. To further understand the role of PQ in the viability and fibrosis, A549 cells were treated with vehicle or different doses of $\mathrm{PQ}$ for the indicated time periods and the cell viability and levels of FN and COL-I expression were determined. As shown in Figure 4(a), treatment with different doses of $\mathrm{PQ}$ reduced the viability of A549 cells in a dose- and time-dependent manner. The levels of FN and COL-1 expression increased in A549 cells in a dose-dependent manner (Figure 4(b)). Flow cytometry analysis revealed that PQ exposure decreased the MMP in A549 cells (Figure 4(c)). Western blot analysis indicated that the relative levels of PINK1 and Parkin expression in the PQ-exposed cells were significantly higher than those in the control cells, and the effects of PQ on enhancing PINK1 and Parkin expression tended to be dosedependent $(p<0.05$ for all, Figure $4(d))$. Such data indicated that PQ exposure reduced the viability and stimulated profibrogenic factor expression in A549 cells by enhancing PINK1 and Parkin expression and mitophagy.

3.3. PINK1 Silencing Mitigates the PQ-Increased FN, COL-I, and Parkin Expression in A549 Cells. Finally, we examined the effect of PINK1 silencing on the levels of FN, COL-I, and Parkin expression in A549 cells. We found that transfection with PINK1-specific siRNA, but not the control siRNA, significantly mitigated not only Parkin expression but also attenuated the PQ-up-regulated FN and COL-I expression in A549 cells ( $p<0.05$ for all, Figure 5). Therefore, induction of mitophagy and fibrosis by PQ may be dependent on enhancing the PINK1-related signaling in A549 cells.

\section{Discussion}

PQ is a highly toxic pesticide, and there is currently no specific measure or antidote for evaluation and treatment of PQ toxicity following PQ poisoning. Because PQ acute poisoning can lead to acute multiple organ failure and chronic pulmonary fibrosis and death, the mortality of patients with $\mathrm{PQ}$ poisoning is high [8].

We found that PQ exposure caused ALI and pulmonary fiber accumulation in rats, consistent with previous studies [9]. Evidently, many inflammatory infiltrates, obviously alveolar epithelial edema, telangiectasia, and red blood cell leakage occurred in the lung tissues of rats at $2 \mathrm{~h}$ after PQ exposure, accompanied by collagen fiber accumulation. These pathogenic changes increased with time. Similarly, PQ exposure also significantly reduced the viability of A549 cells and enhanced their expression of FN and COL-I in a doseand time-dependent manner.

Mitophagy is a selective autophagy process and is crucial for the maintenance of mitochondrial function in many types of cells [10]. It is well known that PQ can induce oxidative stress and produce ROS, leading to depletion of antioxidants and development of mitophagy and fibrosis 
$[3,11,12]$. Actually, previous studies have shown that PQ exposure decreased the MMP and increased PINK1 and Parkin expression, leading to mitophagy and apoptosis in A549 cells [13]. In this study, we found that PQ exposure significantly changed the microstructure of mitochondria with typical feature of mitophagosomes and upregulated PINK1 and Parkin expression in the lung of rats. Such novel data suggest that PINK1 may selectively recruit Parkin on the mitochondrial membrane to ubiquitinate mitochondrial proteins [14]. More importantly, PINK1 silencing significantly mitigated the PQ-upregulated FN and COL-I expression in A549 cells. Accordingly, our findings indicated that PQ exposure caused mitophagy in lung epithelial cells by enhancing the PINK1/Parkin signaling in vivo and in vitro. Hence, inhibition of the PINK1/Parkin signaling may be valuable therapeutic strategies for intervention of $P Q$ poisoning.

In summary, our data indicated that PQ exposure induced ALI and progressive fibrosis in the lungs of rats, accompanied by upregulated PINK1 and Parkin expression and mitophagy in the lung cells. Similarly, PQ exposure significantly reduced the viability and MMP, but enhanced FN, COL-I, PINK1, and Parkin expression in A549 cells, which were significantly mitigated by PINK1 silencing. Such data suggest that the PINK1/Parkin signaling may be valuable therapeutic targets for intervention of $P Q$ poisoning.

\section{Data Availability}

The datasets generated and analyzed during the current study are available from the corresponding author on reasonable request.

\section{Conflicts of Interest}

The authors declare no conflicts of interest.

\section{Acknowledgments}

This study was supported by a grant from the Bureau of Science and Technology and Intellectual Property Nanchong City, China (Grant no. 15A0017).

\section{References}

[1] W. Myung, G. H. Lee, H. H. Won et al., "Paraquat prohibition and change in the suicide rate and methods in South Korea," PLoS One, vol. 10, no. 6, Article ID e0128980, 2015.

[2] R. J. Dinis-Oliveira, J. A. Duarte, A. Sánchez-Navarro, F. Remião, M. L. Bastos, and F. Carvalho, "Paraquat poisonings: mechanisms of lung toxicity, clinical features, and treatment," Critical Reviews in Toxicology, vol. 38, no. 1, pp. 13-71, 2008.

[3] Z. Suntres, "Role of antioxidants in paraquat toxicity," Toxicology, vol. 180, no. 1, pp. 65-77, 2002.

[4] X. Shao and J. H. Chen, "Progress on pathogenesis and treatment of paraquat-induced pulmonary fibrosis," Journal of Zhejiang University (Medical Science), vol. 43, no. 6, pp. 717-727, 2014.
[5] J. P. Gray, D. E. Heck, V. Mishin et al., "Paraquat increases cyanide-insensitive respiration in murine lung epithelial cells by activating an $\mathrm{NAD}(\mathrm{P}) \mathrm{H}$ : paraquat oxidoreductase," Journal of Biological Chemistry, vol. 282, no. 11, pp. 7939-7949, 2007.

[6] J. J. Lemasters, "Selective mitochondrial autophagy, or mitophagy, as a targeted defense against oxidative stress, mitochondrial dysfunction, and aging," Rejuvenation Research, vol. 8, no. 1, pp. 3-5, 2005.

[7] S. V. Szapiel, N. A. Elson, J. D. Fulmer, G. W. Hunninghake, and R. G. Crystal, "Bleomycin-induced interstitial pulmonary disease in the nude, athymic mouse," The American Review of Respiratory Disease, vol. 120, no. 4, pp. 893-899, 1979.

[8] I. B. Gawarammana and N. A. Buckley, "Medical management of paraquat ingestion," British Journal of Clinical Pharmacology, vol. 72, no. 5, pp. 745-757, 2011.

[9] A. Bertram, S. S. Haenel, J. Hadem et al., "Tissue concentration of paraquat on day 32 after intoxication and failed bridge to transplantation by extracorporeal membrane oxygenation therapy," BMC Pharmacology and Toxicology, vol. 14, no. 1, p. 45, 2013.

[10] R. J. Youle and D. P. Narendra, "Mechanisms of mitophagy," Nature Reviews Molecular Cell Biology, vol. 12, no. 1, pp. 9-14, 2011.

[11] L. Wei, J. Wang, A. Chen, J. Liu, X. Feng, and L. Shao, "Involvement of PINK1/parkin-mediated mitophagy in $\mathrm{ZnO}$ nanoparticle-induced toxicity in BV-2 cells," International Journal of Nanomedicine, vol. 12, pp. 1891-1903, 2017.

[12] X. Wei, Y. Qi, X. Zhang et al., "Cadmium induces mitophagy through ROS-mediated PINK1/Parkin pathway," Toxicology Mechanisms and Methods, vol. 24, no. 7, pp. 504-511, 2014.

[13] D.-Z. Sun, C.-Q. Song, Y.-M. Xu et al., "Involvement of PINK1/Parkin-mediated mitophagy in paraquat- induced apoptosis in human lung epithelial-like A549 cells," Toxicology in Vitro, vol. 53, pp. 148-159, 2018.

[14] S. Rodriguez-Enriquez, I. Kim, R. T. Currin, and J. J. Lemasters, "Tracker dyes to probe mitochondrial autophagy (mitophagy) in rat hepatocytes," Autophagy, vol. 2, no. 1, pp. 39-46, 2006. 


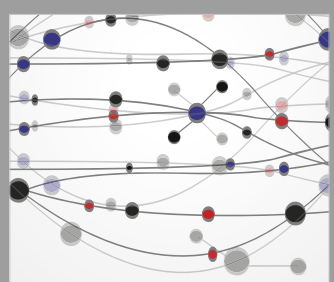

The Scientific World Journal
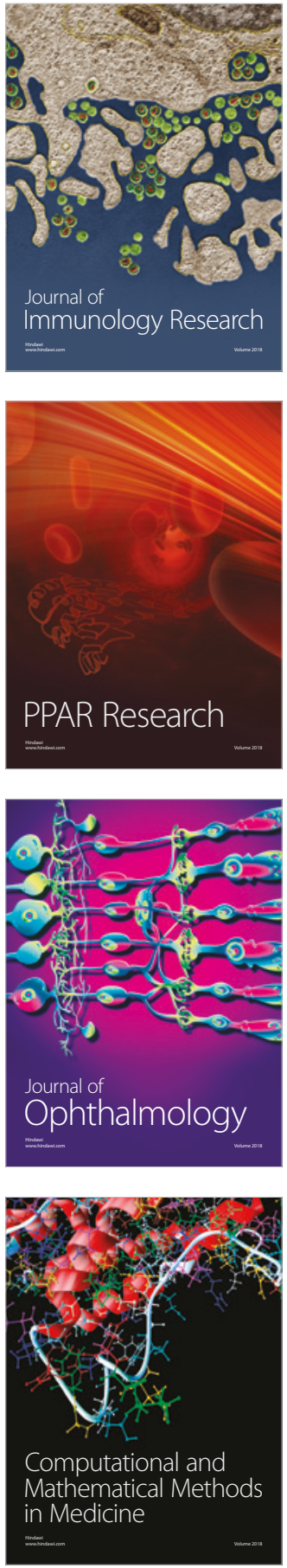

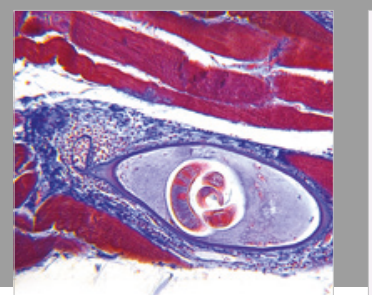

Gastroenterology Research and Practice

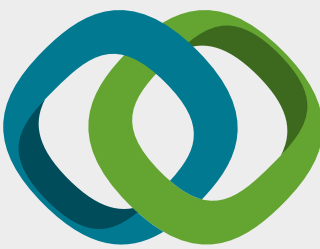

\section{Hindawi}

Submit your manuscripts at

www.hindawi.com
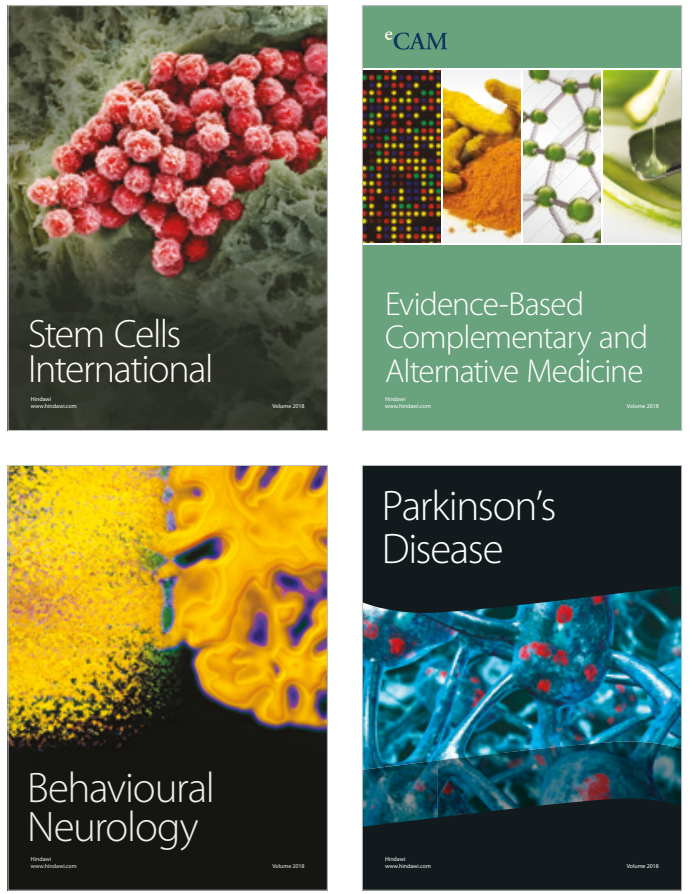

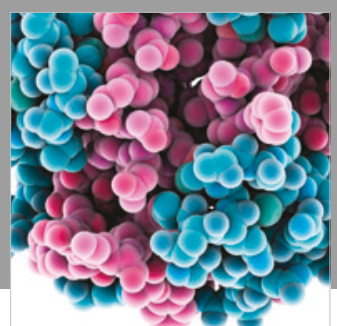

ournal of

Diabetes Research

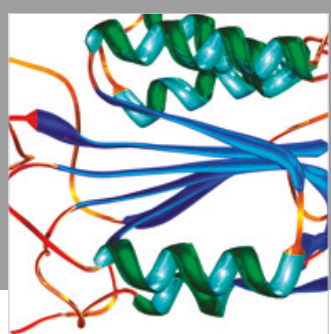

Disease Markers
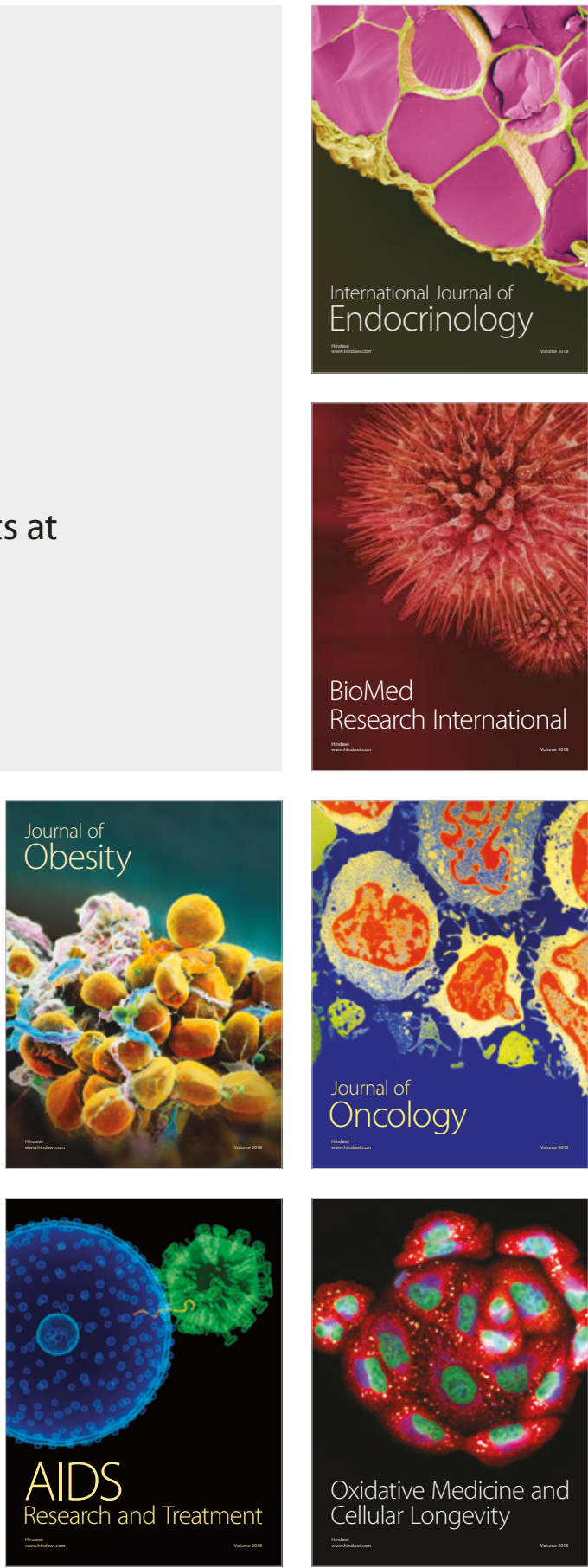